\title{
Feeding pregnant rats a protein-restricted diet persistently alters the methylation of specific cytosines in the hepatic PPAR $\alpha$ promoter of the offspring
}

\author{
Karen A. Lillycrop ${ }^{1}$, Emma S. Phillips ${ }^{1}$, Christopher Torrens ${ }^{2}$, Mark A. Hanson ${ }^{2}$, Alan A. Jackson ${ }^{3}$ \\ and Graham C. Burdge ${ }^{4 *}$ \\ ${ }^{1}$ Development and Cell Biology, Biomedical Sciences Building, University of Southampton, Bassett Crescent East, Southampton \\ SO16 7PX, UK \\ ${ }^{2}$ Institute of Developmental Sciences Building, Centre for DOHaD, Southampton General Hospital, Tremona Road, Southampton \\ SO16 6YD, UK \\ ${ }^{3}$ Institute of Human Nutrition, Southampton General Hospital, Tremona Road, Southampton SO16 6YD, UK \\ ${ }^{4}$ Institute of Human Nutrition, Institute of Developmental Sciences Building, Southampton General Hospital, Tremona Road, \\ Southampton SO16 6YD, UK
}

(Received 28 March 2007 - Revised 19 November 2007 - Accepted 20 November 2007 - First published online 11 January 2008)

Induction of an altered phenotype by prenatal under-nutrition involves changes in the epigenetic regulation of specific genes. We investigated the effect of feeding pregnant rats a protein-restricted (PR) diet with different amounts of folic acid on the methylation of individual CpG dinucleotides in the hepatic PPAR $\alpha$ promoter in juvenile offspring, and the effect of the maternal PR diet on CpG methylation in adult offspring. Pregnant rats (five per group) were fed $180 \mathrm{~g} / \mathrm{kg}$ casein (control) or $90 \mathrm{~g} / \mathrm{kg}$ casein with $1 \mathrm{mg} / \mathrm{kg}$ folic acid (PR), or $90 \mathrm{~g} / \mathrm{kg}$ casein and $5 \mathrm{mg} / \mathrm{kg}$ folic acid (PRF). Offspring were killed on postnatal day 34 (five males and females per group) and day 80 (five males per group). Methylation of sixteen CpG dinucleotides in the PPAR $\alpha$ promoter was measured by pyrosequencing. Mean PPAR $\alpha$ promoter methylation in the PR offspring (4.5\%) was $26 \%$ lower than controls $(6.1 \%)$ due to specific reduction at $\mathrm{CpG}$ dinucleotides $2(40 \%), 3(43 \%), 4(33 \%)$ and $16(48 \%)(P<0.05)$. There was no significant difference in methylation at these $\mathrm{CpG}$ between control and PRF offspring. Methylation of CpG 5 and 8 was higher (47 and $63 \%$, respectively, $P<0.05$ ) in the PRF offspring than control or PR offspring. The methylation pattern in day 80 PR offspring was comparable to day $34 \mathrm{PR}$ offspring. These data show for the first time that prenatal nutrition induces differential changes to the methylation of individual $\mathrm{CpG}$ dinucleotides in juvenile rats which persist in adults.

Fetal programming: Epigenetic regulation: Rats: PPAR $\alpha$

There is an increasing awareness that aspects of the prenatal environment, including nutrition, provide cues which act through developmental plasticity to alter the phenotype of the offspring ${ }^{(1)}$. In man, such developmental cues contribute to the early origins of risk of chronic diseases ${ }^{(2)}$. In rats, variations in the phenotype of the offspring are induced by feeding pregnant dams a diet with a moderate reduction in $\operatorname{protein}^{(3)}$. Induced changes to the phenotype which persist throughout the life-span involve stable alterations to the expression of the genome ${ }^{(4)}$. Epigenetic regulation of genes, specifically methylation of clusters of $\mathrm{CpG}$ dinucleotides (islands) and covalent modifications of histones in promoter regions, are established during early life and confer stable silencing of transcription which is critical for cell differentiation ${ }^{(5)}$. We have shown that feeding pregnant rats a protein-restricted (PR) diet during pregnancy increased glucocorticoid receptor (GR) and PPAR $\alpha$ expression in the liver of the offspring by inducing hypomethylation of their respective promoters, possibly by decreasing DNA methyltransferase- 1 expression, and increasing levels of transcription-permissive histone modifications ${ }^{(6-8)}$. Hypomethylation and the resulting increase in GR and PPAR $\alpha$ expression was prevented by increasing the folic acid content of the PR $\operatorname{diet}^{(7)}$. In human umbilical cord, DNA methyltransferase-1, but not DNA methyltransferase-3a, expression was positively associated with methylation of the GR promoter ${ }^{(8)}$. Overall, these findings suggest an epigenetic mechanism by which prenatal nutrition may induce an altered phenotype in the offspring.

Abbreviations: GR, glucocorticoid receptor; PR diet, protein-restricted diet (90 g/kg casein and $1 \mathrm{mg} / \mathrm{kg}$ folic acid); PRF diet, protein-restricted diet with increased folic acid $(90 \mathrm{~g} / \mathrm{kg}$ casein and $5 \mathrm{mg} / \mathrm{kg}$ folic acid).

* Corresponding author: Dr G. C. Burdge, fax +44 238079 5255, email g.c.burdge@ soton.ac.uk 
The promoter regions of many genes contain several $\mathrm{CpG}$ dinucleotides at which induced changes in methylation, and hence epigenetic regulation, might occur. For example, increased pup licking and grooming by lactating rats decreased stress response in the adult offspring by increasing GR expression in the hippocampus through differential hypomethylation of individual $\mathrm{CpG}$ dinucleotides in the GR promoter, leading to the altered binding of transcription factors ${ }^{(9-11)}$. One example is the binding of NF1A which was regulated by the methylation of a single cytosine ${ }^{(9-11)}$. However, it is not known whether maternal under-nutrition during pregnancy alters the methylation of specific $\mathrm{CpG}$ dinucleotides within a gene promoter or if all $\mathrm{CpG}$ are affected to the same extent. Furthermore, it is not known whether such patterns of methylation, once induced, are permanent. We have investigated the effect of feeding pregnant rats a PR diet on the methylation of individual $\mathrm{CpG}$ dinucleotides in the PPAR $\alpha$ promoter in the liver, in both juvenile and adult offspring. We also investigated the effect of increasing the folic acid content of the PR diet on $\mathrm{CpG}$ methylation of the PPAR $\alpha$ promoter in juvenile offspring.

\section{Materials and methods}

\section{Animal procedures}

In order to validate assessment of promoter methylation by methylation-sensitive RT-PCR which we have used previously ${ }^{(7,12)}$, the livers studied here were from the same rats. The sample size at both ages was sufficient to detect significant differences in promoter methylation between dietary groups $^{(7,12)}$. All animal procedures were conducted in accordance with the UK Home Office Animals (Scientific Procedures) Act (1986). Briefly, Virgin Wistar rats (five per dietary group) were fed from conception to delivery with isoenergetic diets containing either $180 \mathrm{~g} / \mathrm{kg}$ casein and $1 \mathrm{mg} / \mathrm{kg}$ folic acid (control), $90 \mathrm{~g} / \mathrm{kg}$ casein and $1 \mathrm{mg} / \mathrm{kg}$ folic acid (PR) or $90 \mathrm{~g} / \mathrm{kg}$ casein and $5 \mathrm{mg} / \mathrm{kg}$ folic acid (PRF). The composition of the diets has been described previously ${ }^{(7)}$. Dams were fed a standard semi-purified diet (AIN 76A; SDS Ltd) from delivery ${ }^{(7)}$. Litters were reduced to eight at birth, equal numbers of males and females, and offspring were weaned on to AIN 76A at day 28 and were killed at day 34 (juvenile; one liver from each litter was selected for analysis, male to female 3:2) or day 80 (adult; males only, one liver from each litter was selected for analysis, five per group) days. Livers were excised immediately, frozen in liquid nitrogen and stored at $-80^{\circ} \mathrm{C}$.

Analysis of the methylation status of individual $C p G$ dinucleotides in the hepatic PPAR $\alpha$ promoter and PPAR $\alpha$ $m R N A$ expression

Genomic DNA was prepared as described ${ }^{(7)}$ and bisulphite conversion was carried out using the EZ DNA methylation kit (ZymoResearch). The pyrosequencing reaction was carried out by Biotage. Modified DNA was amplified using hot start Taq DNA polymerase (Qiagen) with the following primers: forward GGGGTGTGTTTAGTTTTGAAT, reverse TCACCCCTATCCTAAAACC. PCR products were immobilised on streptavidin-sepharose beads (Amersham), washed, denatured and released into annealing buffer containing the sequencing primer GGGATTTAGTAGGGGA (Biotage). Pyroseqeuncing was carried out using the SQA kit on a PSQ 96MA machine (Biotage) and the percentage methylation was calculated using the beta version of the Pyro Q CpG software from Biotage. Assay precision was between 0.8 and $1.8 \%$ standard deviation and detection limits were $2-5 \%$ methylation. The location of the $\mathrm{CpG}$ island in the PPAR $\alpha$ promoter is shown in Fig. 1 (a) and the sequence in Fig. 1 (b). Putative transcription factor binding sites were deduced using Genomatix Matinspector (Genomatix Software $\mathrm{GmbH}$ ).

In order to assess the extent to which the methylation status of individual $\mathrm{CpG}$ is associated with PPAR $\alpha$ mRNA expression we carried out correlation analysis using data published previously where details of the analytical methods are described ${ }^{(7,12)}$.

\section{Statistical analysis}

Values are means and standard deviations of the methylation for individual $\mathrm{CpG}$ dinucleotides expressed relative to the control offspring. For the three groups of offspring studied at $34 \mathrm{~d}$, values within each $\mathrm{CpG}$ were compared by one-way ANOVA with Dunnett's post hoc analysis. For the two groups of male offspring studied at $80 \mathrm{~d}$, statistical comparisons were by Student's unpaired $t$ test. Calculation of Pearson's correlation coefficient was used to determine the relationship between methylation status of individual $\mathrm{CpG}$ in the PPAR $\alpha$ promoter and mRNA expression. Results were combined irrespective of age or maternal diet in order to provide sufficient data-points for correlation analysis.

\section{Results}

The results of analysis of the methylation status of the PPAR $\alpha$ promoter at day 34 are summarised in Fig. 1 (c). One-way ANOVA showed that there were significant differences in methylation between maternal dietary groups at $\mathrm{CpG} 2,3,4$, 5,8 and $16(P<0 \cdot 05)$ and a non-significant trend $(P<0 \cdot 1)$ at $\mathrm{CpG}$ 7, 10 and 11. Mean methylation of the PPAR $\alpha$ promoter in offspring of the dams fed a PR diet during pregnancy $(4.5 \%)$ was significantly lower $(26 \%, P<0.05)$ than controls $(6.1 \%)$ due to selective reduction in methylation $(P<0.05)$ at CpG $2(40 \%), 3(43 \%), 4$ (33\%) and 16 (48\%). There was no significant difference in the methylation of these $\mathrm{CpG}$ dinucleotides between control and PRF offspring. There was a non-significant trend $(P<0 \cdot 1)$ towards lower methylation at $\mathrm{CpG} 7,10$ and 11 in the offspring of the PR $v$. control dams, but not in the offspring of dams fed the PRF diet. Although there was no significant difference in methylation at CpG 5 and 8 between offspring of control and PR dams, methylation was significantly higher $(P<0.05)$ in the offspring of the dams fed the PRF diet compared to controls (47 and $63 \%$, respectively).

There was no significant difference in the mean level or CpG-specific methylation in the PPAR $\alpha$ promoter between offspring of control dams at days 34 and 80 (Fig. 1 (c), (d)). Mean promoter methylation was $28 \%(P<0.05)$ lower in the day 80 PR offspring $(5.1 \%)$ compared to controls $(7 \cdot 1 \%)$. The methylation status of specific $\mathrm{CpG}$ dinucleotides in the hepatic PPAR $\alpha$ promoter was significantly lower in the offspring of PR dams (CpG 2, 72\%; 3, $16 \% ; 4,23 \% ; 7,42 \%$; 
(a)

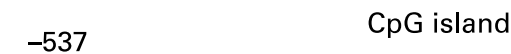
$+259$

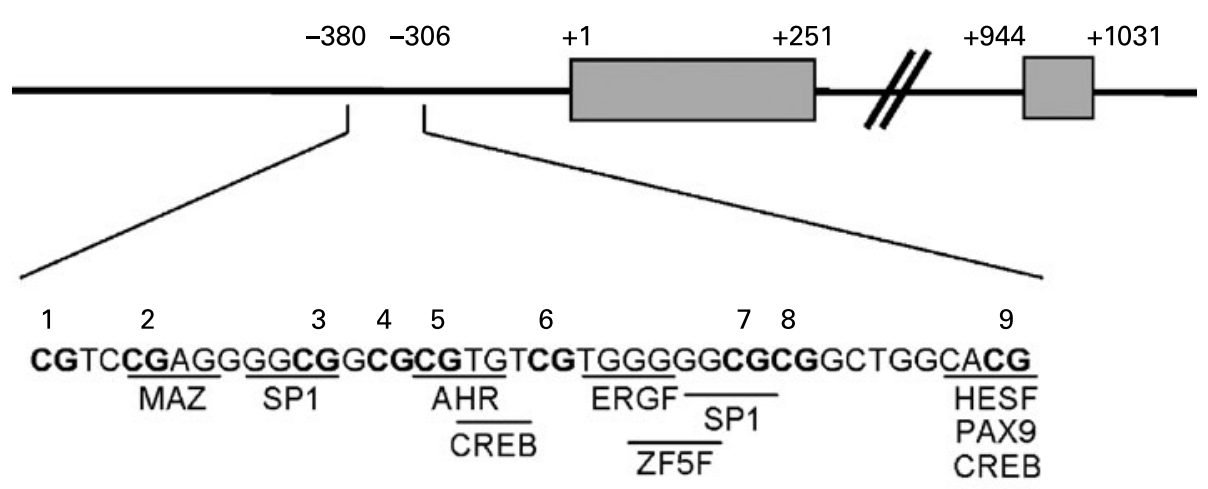

(b)

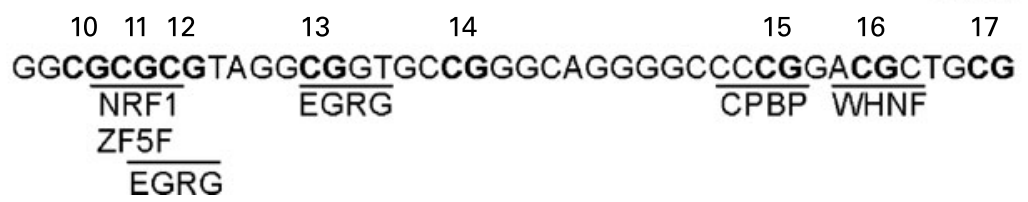

(c)

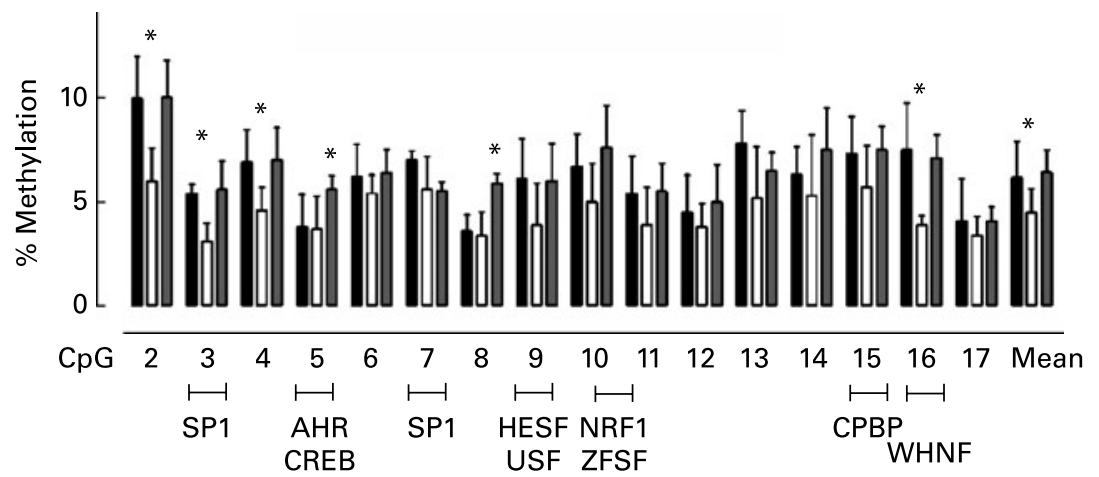

(d)

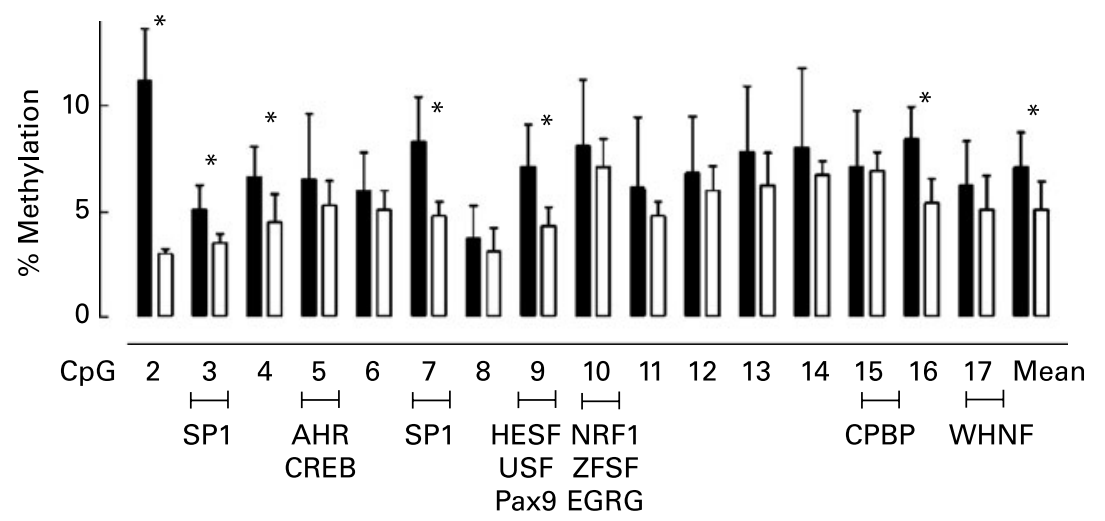

Fig. 1. (a), Structure of the PPAR $\alpha$ gene (www.ensembl.org, gene identity number ENSRNOG00000021463). The location of the CpG island was identified using Methprimer (www.urogene.org/methprimer). (b), Nucleotide sequence of the CpG island showing individual CpG dinucleotides and putative transcription factor binding sites. (c, d), Methylation of individual CpG dinucleotides in the PPAR $\alpha$ promoter in the liver of the offspring of rats fed either a control ( $\square$ ), protein-restricted $(\square)$ or protein-restricted with increased folic acid content ( $\square$ ) diet during pregnancy. Values are means with standard deviations depicted by vertical bars. (c), Day 34 male and female offspring (five per group). Mean values were significantly different from those of the control group (one-way ANOVA with Dunnett's post hoc test): ${ }^{*} P<0.05$. (d), Day 80 male offspring (five per group). Mean values were significantly different from those of the control group (Student's unpaired $t$ test): ${ }^{\star} P<0.05$. AHR, aryl hydrocarbon receptor; CPBP, core promoter-binding protein; CREB, cAMP responsive element binding protein; EGRG, Wilms tumour factor; HESF, hey-like transcriptional repressor; MAZ, Myc-associated zinc finger protein; NRF1, nuclear respiratory factor 1; SP1, specificity protein 1; USF, upstream stimulatory factor; WHNF, winged helix protein; ZF5F, zinc finger domain transcription factor. 
$9,39 \% ; 16,51 \% ; P<0.05)$ compared to the offspring of the control dams (Fig. 1 (d)). Mean difference between offspring of control and PR dams in methylation across all $\mathrm{CpG}$ measured was $39 \%$.

We have published previously the level of PPAR $\alpha$ mRNA expression in these samples ${ }^{(7)}$. There was a significant negative relationship between the methylation status of $\mathrm{CpG} 3$ and 16 (both $P<0.05$ ) such that variation in the level of methylation at $\mathrm{CpG} 3$ predicted $43 \%$ and at $\mathrm{CpG} 16$ predicted $39 \%$ of the difference between individuals in PPAR $\alpha$ mRNA expression.

\section{Discussion}

The results of the present study show for the first time that feeding a PR diet to pregnant rats induces hypomethylation of specific $\mathrm{CpG}$ dinucleotides in the hepatic PPAR $\alpha$ promoter in juvenile offspring. This pattern of cytosine hypomethylation was also found in adult male offspring. In day 34 offspring, the PRF diet prevented hypomethylation of these CpG dinucleotides, but induced hypermethylation of two other CpG.

The present findings agree with our previous data which showed using methylation-specific RT-PCR that feeding a PR diet to pregnant rats induced an overall $26 \%$ reduction in methylation of the PPAR $\alpha$ promoter in the liver of the juvenile and adult offspring ${ }^{(7,12)}$. The level of methylation within the PPAR $\alpha$ promoter region was low (4-10\%) compared to that reported by Weaver et al. ${ }^{(11)}$ for GR in the hippocampus $(14-58 \%)$. However, we did find significant differences in the level of methylation of individual $\mathrm{CpG}$ between the control and PR offspring. Moreover, absolute levels of methylation of specific $\mathrm{CpG}$ dinucleotides in the PPAR $\alpha$ promoter had a significant negative relationship with mRNA expression. This suggests the magnitude of variation in $\mathrm{CpG}$ methylation was sufficient to alter transcription ${ }^{(7)}$ and is consistent with the suggestion that variations in relatively low levels of methylation, compared to imprinted genes, allow fine control of transcription by changing the balance in transcription factor regulation ${ }^{(13)}$. Such subtle changes in epigenetic regulation are more consistent with the graded changes in phenotype induced by the early life environment than the gross phenotypic changes caused by large changes in the methylation of imprinted genes.

The CpG dinucleotides in the PPAR $\alpha$ promoter coincided with the putative binding sites of a number of transcription factors which have an important regulatory role in a wide range of cellular processes (Fig. 1 (b)). It is possible that the differences in methylation of specific $\mathrm{CpG}$ dinucleotides observed may change the regulation of transcription in response to individual transcription factors and consequently the capacity of the tissue to respond to a metabolic challenge. Interestingly, the two $\mathrm{CpG}$ which showed an association between methylation and mRNA expression are located within putative binding sites for the transcription factors specificity protein 1 and winged helix protein, respectively. However, the transcription factor binding sites in Fig. 1 (b) are illustrative and the precise effect of differences in $\mathrm{CpG}$ methylation between offspring from different maternal dietary groups on transcription factor binding awaits experimental investigation.

The present findings agree in both direction and overall magnitude of effect with our previous data which showed, using methylation-specific RT-PCR, that feeding a PR diet to preg- nant rats induced overall hypomethylation of the PPAR $\alpha$ promoter in the liver of the juvenile and adult offspring ${ }^{(7,12)}$. The present data also show that prenatal under-nutrition induced hypomethylation of specific $\mathrm{CpG}$ dinucleotides, rather than altering the methylation of all $\mathrm{CpG}$ in the PPAR $\alpha$ promoter. This is in agreement with the effects of maternal nursing behaviour on the epigenetic regulation of $\mathrm{GR}^{(11)}$. Together these studies suggest that very different aspects of the early life environment induce highly specific changes to the epigenotype of the offspring.

Feeding the PRF diet to pregnant rats prevented hypomethylation of $\mathrm{CpG}$ which showed reduced methylation in the PR offspring, which is consistent with our previous findings ${ }^{(7)}$. However, CpG 5 and 8 were hypermethylated in the PRF offspring which suggests that there may be subtle effects of increased maternal folic acid intake which were not detected by overall assessment of promoter methylation, but which may still alter gene function.

DNA methyltransferase-1 expression and binding of specific transcription factors is reduced in the liver of the offspring of dams fed a PR diet during pregnancy, while this is prevented by increasing the folic acid content of the PR diet $^{(8)}$. Since DNA methyltransferase-1 is targeted to specific genes $^{(14)}$ and $\mathrm{CpG}$ dinucleotides ${ }^{(15)}$, altered DNA methyltransferase-1 expression may provide a mechanism for induction of hypomethylation of specific genes and individual $\mathrm{CpG}$, although how such targeting may occur is not known.

One key principle of the developmental origins of disease hypothesis is that phenotypic characteristics induced in early life persist into adulthood ${ }^{(2)}$. We have shown that overall changes in the methylation of the GR and PPAR $\alpha$ promoters persist in adult offspring ${ }^{(12)}$. The findings of the present study show that the pattern of methylation of specific $\mathrm{CpG}$ dinucleotides in the hepatic PPAR $\alpha$ promoter in adult offspring of PR dams compared to controls were essentially the same as in juvenile offspring. This suggests that the pattern of methylation of individual $\mathrm{CpG}$ induced in early life persists into adulthood.

Together these results show that nutrient constraint before birth induces persistent $\mathrm{CpG}$-specific changes to the epigenetic regulation of the PPAR $\alpha$ promoter and that these changes are associated with altered mRNA expression. If this were to occur in man, the precise pattern of $\mathrm{CpG}$ methylation may provide one mechanism for graded differences in phenotype induced by the developmental environment which result in differential risk of disease.

\section{Acknowledgements}

G. C. B., K. A. L. and M. A. H. designed the study. E. S. P., K. A. L. and C. T. carried out the experimental work. G. C. B., K. A. L. and M. A. H. wrote the paper with contributions from the other authors. G. C. B. and M. A. H. receive salary support from the British Heart Foundation who also funded part of the study. The remainder of the financial support was from departmental funds. There are no conflicts of interest.

\section{References}

1. Bateson $\mathrm{P}$, Barker D, Clutton-Brock T, Deb D, D'Udine B, Foley RA, Gluckman P, Godfrey K, Kirkwood T, Lahr MM, McNamara J, Metcalfe NB, Monaghan P, Spencer HG \& 
Sultan SE (2004) Developmental plasticity and human health. Nature 430, 419-421.

2. Godfrey KM \& Barker DJ (2001) Fetal programming and adult health. Public Health Nutr 4, 611-624.

3. Langley SC \& Jackson AA (1994) Increased systolic blood pressure in adult rats induced by fetal exposure to maternal low protein diets. Clin Sci (Lond) 86, 217-222.

4. Burdge GC, Hanson MA, Slater-Jefferies JL \& Lillycrop KA (2007) Epigenetic regulation of transcription: a mechanism for inducing variations in phenotype (fetal programming) by differences in nutrition during early life? Br J Nutr 97, $1036-1046$.

5. Bird A (2001) DNA methylation patterns and epigenetic memory. Genes Dev 16, 6-21.

6. Burdge GC, Phillips ES, Dunn RL, Jackson AA \& Lillycrop KA (2004) Effect of reduced maternal protein consumption during pregnancy in the rat on plasma lipid concentrations and expression of peroxisomal proliferator-activated receptors in the liver and adipose tissue of the offspring. Nutr Res 24, 639-646.

7. Lillycrop KA, Phillips ES, Jackson AA, Hanson MA \& Burdge GC (2005) Dietary protein restriction of pregnant rats induces and folic acid supplementation prevents epigenetic modification of hepatic gene expression in the offspring. J Nutr 135, $1382-1386$.

8. Lillycrop KA, Slater-Jefferies JL, Hanson MA, Godfrey KM, Jackson AA \& Burdge GC (2007) Induction of altered epigenetic regulation of the hepatic glucocorticoid receptor in the offspring of rats fed a protein-restricted diet during pregnancy suggests that reduced DNA methyltransferase-1 expression is involved in impaired DNA methylation and changes in histone modifications. Br J Nutr 97, 1064-1073.

9. Liu D, Diorio J, Tannenbaum B, Caldji C, Francis D, Freedman A, Sharma S, Pearson D, Plotsky PM \& Meaney MJ (1997) Maternal care, hippocampal glucocorticoid receptors, and hypothalamic-pituitary-adrenal responses to stress. Science 277, $1659-1662$.

10. Francis D, Diorio J, Liu D \& Meaney MJ (1999) Nongenomic transmission across generations of maternal behavior and stress responses in the rat. Science 286, 1155-1158.

11. Weaver IC, Cervoni N, Champagne FA, D'Alessio AC, Sharma S, Seckl JR, Dymov S, Szyf M \& Meaney MJ (2004) Epigenetic programming by maternal behavior. Nat Neurosci 7, 847-854.

12. Burdge GC, Slater-Jefferies JL, Torrens C, Phillips ES, Hanson MA \& Lillycrop KA (2007) Dietary protein restriction of pregnant rats in the $\mathrm{F}_{0}$ generation induces altered methylation of hepatic gene promoters in the adult male offspring in the $F_{1}$ and $\mathrm{F}_{2}$ generations. Br J Nutr 97, 435-439.

13. Mühlisch J, Bajanowski T, Rickert $\mathrm{CH}$, Roggendorf $\mathrm{W}$, Würthwein G, Jürgens H \& Frühwald MC (2007) Frequent but borderline methylation of p16 (INK4a) and TIMP3 in medulloblastoma and sPNET revealed by quantitative analyses. J Neurooncol 83, 17-29.

14. Robertson KD, Ait-Si-Ali S, Yokochi T, Wade PA, Jones PL \& Wolffe AP (2000) Dnmt1 forms a complex with Rb, E2F1 and $\mathrm{HDAC} 1$ and represses transcription from $\mathrm{E}_{2} \mathrm{~F}$-responsive promoters. Nat Genet 25, 338-342.

15. Valinluck V \& Sowers LC (2007) Endogenous cytosine damage products alter the site selectivity of human DNA maintenance methyltransferase DNMT1. Cancer Res 67, 946-950. 